\title{
Deux modèles métaphysiques de la théorie littéraire
}

\section{Patrick Marot}

Université de Toulouse 2 - Jean Jaurès*

La critique littéraire moderne et contemporaine est tributaire de modèles métaphysiques antagonistes : la modernité se déploie en effet à partir de la conception romantique du « système », qui commande les approches herméneutiques. Deux inflexions en contestent toutefois le principe ; l'une (la " nouvelle critique ») qui radicalise le système en « extème »; l'autre qui par réaction tente une refondation onto-théologique.

Mots-clés : extème, herméneutique, métaphysique, modèle, onto-théologie, poétique, post-structuralisme, système, théologico-politique, théorie.

La théorie littéraire, au sens que nous lui donnons aujourd'hui de réflexion sur la spécificité du littéraire à travers la diversité de ses expressions historiques, apparaît au moment même où s'effectue la mise au tombeau des métaphysiques classiques sous les attaques du criticisme kantien et fichtéen - à la charnière des $\mathrm{xviii}^{\mathrm{e}}$ et $\mathrm{xix}^{\mathrm{e}}$ siècles, et où simultanément se mettent en place avec l'idéalisme allemand des projets esthétiques susceptibles d'instaurer à nouveau frais les conditions d'une union retrouvée du sujet et du monde. L'exégèse, avec Schleiermacher ${ }^{1}$ dont le nom doit être associé à celui de Friedrich Schlegel dans l'invention de la poétique du romantisme d'Iéna (voir Schleiermacher 1987), a pris après Johann Georg Hamann la mesure de l'impossibilité d'un accès direct au Verbe divin (voir Hamann 2002), et par là-même de l'illusion que pouvait constituer l'idée d'une traduction directe par le langage d'un quelconque sens du monde. C'est, rappelonsle, l'époque où Jean-Paul Richter à la suite de la lettre ouverte de Jacobi à Fichte (Jacobi 1946 : 303-335) s'interroge dans sa Clavis fichteana (Richter 1800) sur l'abîme qu'ouvre devant les écrivains la perte d'une continuité entre les essences

\footnotetext{
"patmarot@orange.fr

${ }^{1}$ S'il est l'auteur d'une Esthétique, d'une Éthique et d'une Dialectique, Friedrich Schleiermacher est surtout connu comme fondateur de l'herméneutique moderne, dont il réserve toutefois l'exercice à l'exégèse biblique. Voir Herméneutique. Pour une logique du discours individuel, Paris, Le Cerf, 1987.
} 
et les phénomènes, et la libération aussi exaltante qu'inquiétante qui s'ensuit des puissances de l'imagination qu'au demeurant il s'ingénia plus que tout autre à cultiver. La théorie littéraire, en tant qu'elle interroge les conditions de possibilité et les modalités pour la littérature d'une expression du sens, est indissociable d'un questionnement métaphysique dont elle accompagne les bouleversements depuis le moment criticiste jusqu'à nos jours, et dont elle nourrit - souvent de manière explicite - les différents modèles qu'elle propose pour penser ce qui se pense sous le terme de « littérature » ou à travers la contestation de celui-ci.

Un troisième terme intervient dans ce débat, et qui en est également indissociable : celui d'une réflexion sur l'origine et le fonctionnement des langues, enquête philologique dont l'essor est concomitant avec l'affaiblissement de la rhétorique, et manifeste le déplacement de l'attention du transcendant qui fondait cette dernière depuis Aristote et Porphyre vers le transcendantal, le fondement identitaire des sujets étant désormais à trouver dans une caractérisation immanente, relative et historique dont les enquêtes sur les langues primitives proposées par Le Monde primitif de Court de Gébelin (Court de Gébelin 1773-1782) ou l'Essai sur l'origine des langues de Rousseau (Rousseau 1781), par exemple, avaient contribué à ouvrir la carrière. La réflexion de Diderot dans sa « Lettre sur les sourds et muets (Diderot 1978) » désigne de manière aussi prophétique que programmatique ce qui se tient désormais à la fois à l'origine et à l'horizon du langage comme de ce qu'il appelle "poésie », (et qui prendra bientôt le nom de « littérature ») : une énergie transcendant non seulement le discours ordinaire mais aussi l'éloquence, dont le sens reste dans l'inaccessible, et qu'il qualifie du terme d' « hiéroglyphes » (idem : 172) dont chacun sait qu'ils étaient jusqu'à Champollion des signes indéchiffrables. Ce qui fait le fond des langues comme celui de la littérature assigne l'homme à devoir déchiffrer le sens de son expérience dans la confrontation à une dynamique énigmatique qui tient lieu de fondement paradoxal, et qui assimile désormais l'être au devenir. Les recherches sur une hypothétique langue originelle, qui fonde par exemple les travaux philologiques d'un Schlegel sur le sanskrit (Schlegel 1813) ou d'un Bopp sur la linguistique comparative (Bopp 2007), disent en effet la dualité d'un fondement perdu dont la quête - celle d'une identité qui soit indissociablement une origine et un projet - conçoit désormais le sens comme une construction toujours à reprendre et non comme un socle.

Les concepts mêmes de littérature et de langue relèvent en l'occurrence d'un dispositif métaphysique qui prend acte de cette dérobade du fondement, de cette bascule de l'être comme du monde et du sujet dans la dynamique processuelle du devenir. Le philosophe Pierre Caye décrit ce dispositif comme celui du « système ${ }^{2}$ », c'est-à-dire d'une « articulation triadique » de ces trois instances que

\footnotetext{
2 Le terme de « système » dans son sens spéculatif moderne apparaît dans l'écrit Le plus ancien programme de l'idéalisme allemand (attribué à Friedrich Schelling mais probablement conçu avec Hegel et Hölderlin qui étaient ses compagnons de chambre au Stift de Tübingen), 1795. Je renvoie
} 
sont l'homme, le monde et l'être, et dont l'histoire va de l'idéalisme allemand à Heidegger (Caye 2015 : 85). Ce dernier qualifie l'opposition de la métaphysique classique et de ce qui s'ouvre avec l'idéalisme moderne comme celle de l' « ontothéologie » et du «théologico-politique $»^{3}$. Il y a là le souci d'une réparation de la fracture sémiotique ouverte par le criticisme kantien - une réparation au sein même de l'ordre complexe et médiatisé des phénomènes, et dont Hamann, ce génial penseur néoplatonicien qui fut l'ami et le contradicteur intime de Kant à Königsberg et qui influença fortement Herder, Goethe, Schleiermacher et quelques autres, constitue un maillon privilégié (voir supra, note 1). Cette métaphysique de la modernité est le système et la philosophie qui conviennent à un Occident qui se pense désormais comme procès en pleine expansion ; un Occident qui ne peut plus se satisfaire des métaphysiques du fondement qui avaient prévalu jusqu'alors et dont les Lumières avaient érodé l'édifice jusqu'au coup de grâce donnée par Kant et par Fichte; un Occident qui entend son histoire comme un accroissement continu de puissance, et à qui le capitalisme fournit la réponse socio-économique qui convient ; un Occident enfin qui a donné à l'homme, à travers les principes d'égalité, de liberté et d'individualité, une nouvelle définition anthropologique dont la projection littéraire est une redéfinition de la notion d'œuvre - elle-même entendue comme singulière, autonome et expression d'une liberté créatrice.

Ce qui caractérise le « système » compris, ainsi que le rappelle Heidegger dans son Schelling, comme "système de la liberté » (Heidegger 1977 : 46) est la différenciation, l'égalité et la mobilité de ces trois instances. On se rappelle que Stendhal, dans son Racine et Shakespeare, a mis en évidence cette nouvelle caractérisation d'un sujet que Molière ne fait plus rire parce qu'il a été délivré des carcans sociaux et religieux par la Révolution, et que l'expérience de la Grande armée l'a rendu l'égal de quiconque (voir Stendhal 1823-1825, chap. 2). Chez Balzac de même les Rastignac et les Vautrin peuvent désormais affirmer leur destin dans un monde livré à la multitude des appétits individuels et condamné à faire son deuil des principes éternels et sacrés, et de l'harmonie stable et hiérarchique qui en était le corollaire idéologique.

La théorie la plus idoine de cette métaphysique moderne du système est sans doute l'herméneutique. On sait que celle-ci prend un nouvel essor avec la pensée de Schleiermacher, dont l'exégèse biblique renouvelée trouve son pen-

pour sa présentation métaphysique au récent ouvrage de Pierre Caye, Critique de la destruction créatrice (Caye 2015 : 85 sq.).

3 Le concept d' " onto-théologie » apparaît chez Emmanuel Kant dans sa Critique de la raison pure (A632/B660). 11 est repris en particulier par Martin Heidegger dans sa conférence de 1957 Constitution onto-théologique de la métaphysique. Celui de «théologico-politique, issu du Tractatus theologico-politicus de Spinoza, est notamment repris par Heidegger, en opposition avec le premier, dans « Constitution onto-théologique de la métaphysique » (1957), repris dans Questions I-II, Paris, Gallimard, 1968, et dans Schelling. Le traité de 1809 sur l'essence de la liberté humaine, Paris, Gallimard (nrf), 1971. 
dant tant dans la spéculation poéticienne d'un Friedrich Schlegel ${ }^{4}$ que, quelques années plus tard, dans la méthodologie critique d'un Sainte-Beuve (voir Molino 1993 : 196). L'herméneutique en effet pense le sujet et l'œuvre comme des instances non pas identiques mais coextensives, qui se fondent et se révèlent réciproquement, construisant à travers ce procès une relation homogène à l'être. Le parcours du divers et les aléas du devenir sont données par Hans Georg Gadamer, dans la continuité de l'herméneutique romantique de Schleiermacher, comme le chemin même de la quête d'une vérité du sens - chemin constamment décentré par les polarisations du familier et de l'étrangeté, par les décalages de la question et de la réponse, mais recentré par le double postulat d'un achèvement possible de la quête au terme d'une série d'ajustements, et conjointement d'une univocité du sens (voir Gadamer 1996). L'herméneutique négocie ainsi à travers les jeux de convergence entre le sujet et le monde un palliatif à la dérobade du fondement, assurant par là une reconquête de l'être sous les espèces même du devenir.

On conçoit que cette double institution, réciproque et sans antécédence, du sujet et du monde, trouve dans l'œuvre le lieu privilégié de ce qu'on peut appeler une expérience intime de la totalisation. La pensée critique de Jean Starobinski est à cet égard exemplaire, qui cherche la juste " relation critique » entre ce qu'il appelle d'une part la « conscience fabuleuse » (Starobinski $1961: 25)$ qui dissout le lecteur dans l'œuvre - image de la fusion indifférenciatrice et par là-même dangereuse du sujet et du monde, et d'autre part la conscience surplombante qui risque, comme il le remarque, de figer les instances du sujet à propos du style de L'Esprit des lois dans son Montesquieu par lui-même (voir Starobinski 1953), du monde et de l'être au lieu de les faire circuler. Dans un cas comme dans l'autre, l'œuvre en tant qu'œuvre se trouverait menacée soit parce que serait manquée la visée d'une construction du sens à travers l'objectivation de soi, soit parce que serait bloqué la dynamique et l'expansion du devenir.

L'un de ces périls - péril si on se place du point de vue du fonctionnement idéal de la métaphysique du "système »- est incarné par la tentation psychanalytique de l'herméneutique, que Starobinski sait tout à la fois intégrer et tenir à distance tout en faisant la part, comme plus tard un Jean-Pierre Richard, d'une sorte de point aveugle qui fonde les conditions de visibilité du regard moderne, et qu'il repère dans son 1789 (voir Starobinski 1973) à partir de la Révolution française. La même remarque peut être faite à propos des paragrammes et anagrammes de Ferdinand de Saussure, que Starobinski a révélés au public en 1964 et 1971 (voir Leroy 1974 : 659-660 ). Ceux-ci ouvrent en effet dans le langage une sorte de double-fond où pourrait tendanciellement fusionner ce qui est posé comme principe de différenciation au cœur de la structure de la langue par la

\footnotetext{
${ }^{4}$ C'est notablement le cas dans le fameux Fragment 116 de l'Athenaeum (cf. Jean-Luc Nancy et Philippe Lacoue-Labarthe, L'Absolu littéraire, Paris, Seuil, p. 112).
} 
sémiologie saussurienne : une menace latente, ici maintenue dans une marge opérante mais contrôlée, et que voudront pleinement libérer, en assumant le risque de rompre les digues, les théoriciens de la Nouvelle critique. Certes le fondement de l'être, chez Starobinski comme chez maître Eckhart dont la métaphysique du devenir a fait, avec Hegel et Schelling, une de ses références majeures, est « sans fond »; certes le sujet risque d'être happé par son propre inconscient et le monde par son immensité même. Pour autant les trois instances circulent et se refondent par là-même dans l'affirmation de l'œuvre entendue comme espace de liberté et de souveraineté poétique.

L'autre péril est celui du figement de l'être dans une tentation sécularisée du fondement, qui menacerait de gripper le système en en entravant la dynamique libératrice. Les pensées critiques d'Ernst Robert Curtius (Curtius 1956) ou de Hans Robert Jauss (Jauss 1988), qui sont aussi des sommets de l'approche herméneutique de la littérature, ne vont pas à cet égard sans représenter ce qu'on pourrait appeler une tentation onto-théologique de la métaphysique du devenir. On peut noter à cet égard, sans entrer dans les modèles méthodologiques élaborés par ses savants, que ceux-ci se sont efforcés d'unifier le sens en l'inscrivant dans la continuité d'une tradition. Ainsi chez Curtius, la tradition européenne constitue un espace homogène où puissent converger les totalisations universalistes proposées par les grands créateurs (voir Curtius 1956). Son Balzac publié en 1932 (Curtius 1999) montre par exemple comment le secret intime qui unifie la structure complexe de La Comédie humaine entre en résonance avec le projet d'une inscription universaliste de l'expérience humaine dans le monde. La circulation entre le sujet, le monde et l'être est dès lors parfaitement harmonieuse, mais s'enferme - de manière évidemment délibérée - dans la boucle identitaire d'une tradition et d'une continuité dont Curtius rappelle l'urgence face au danger des nationalismes qui ont conduit à la Seconde guerre mondiale. La controverse amicale avec Auerbach souligne a contrario cette tentative chez Curtius de restauration onto-théologique et de stabilisation du fondement au sein même de l'économie du « système » métaphysique moderne : si Mimesis, écrit par Auerbach en exil à Istanbul en 1938 comme un dialogue avec Curtius et Benjamin (voir Auerbach 1969), met également l'accent sur la spécificité d'un rapport au monde propre à la culture européenne, l'auteur met toutefois en évidence la violence d'un modèle culturel homogénéisant et globalisant imposé par les Lumières : celui d'une représentation figurale d'origine chrétienne, où tout événement dans le monde sensible renvoie à une signification spirituelle, et où l'historicité s'abolit dans l'horizon d'un futur qui fonde dans l'éternité le présent et le passé : à ce modèle, l'essai intitulé Figura (Auerbach 1977) oppose la diversité des configurations symboliques de l'Histoire (antique, judaïque, chrétienne, moderne), et par là-même la diversité des modèles représentationnels instituant l'horizon du sens et le rapport du sujet au monde. 
Un autre exemple majeur de cette tentation onto-théologique qui s'est emparée du « système » dans certaines approches herméneutiques est celui de Hans Robert Jauss. Celui-ci, disciple de Gadamer, écrit après la parution des ouvrages fondateurs de la Nouvelle critique, auxquels il entend résister en restaurant un fondement stable au sein même de la logique socio-historique du devenir et de l'institution phénoménologique de l'être et du sens. Jauss reprend de l'auteur de Vérité et méthode (voir Gadamer 1960) l'idée d'une universalité transhistorique du geste interprétatif, que confirme le modèle épistémologique de la fusion des horizons de réception. Gadamer, qui avait théorisé le déplacement, à travers décentrements et médiations, du point de fuite du sens, avait toutefois arrêté celui-ci en substantialisant une sorte de classicisme idéal. Or si Jauss stigmatise chez son maître cette « métaphysique du Beau intemporel », il la restaure non dans l'ordre esthétique mais dans celui de la phénoménologie, par l'achèvement promis, et qu'il entend illustrer dans Pour une herméneutique littéraire (Jauss 1988), d'une univocité du sens des œuvres et d'une continuité unifiante de leur réception à travers les différents socles épistémologiques (antique, médiéval, moderne) successivement proposés par l'Histoire. C'est l'opposition chère à Jauss entre une sémantique variable et une syntaxe stable.

De tels exemples pourraient bien donner raison à Heidegger, qui estimait dans son Schelling que le « système » n'est pas parvenu à rompre vraiment dans son principe avec la logique onto-théologique du fondement (voir Heidegger 1977 : 47). Si Schelling, selon l'auteur d'Etre et temps, est allé plus loin que quiconque dans la libération du " système ", il est toutefois selon lui resté ambigu parce qu'il a maintenu une persistance du fondement incompatible avec l'exigence de liberté qui fonde le système lui-même, et que le philosophe de Marbourg ne conçoit qu'absolue et sans limite. De fait, les différents modèles critiques de l'herméneutique, qui incarnent exemplairement depuis Schleiermacher, Schlegel et tant d'autres la conception métaphysique moderne du « système », montrent que ce maintien relatif du fondement - à travers les manières mêmes de penser une cohérence possible du devenir et du sens - est la condition de l'équilibre entre les trois termes qu'il convient de préserver tout en permettant leur circulation : le sujet libre et autonome, le monde mystérieux mais appropriable, l'être mouvant et dérobé mais garantissant l'horizon du sens. Pierre Caye, déjà cité, met en évidence ce qu'implique le projet de destruction de la métaphysique pour Heidegger (Caye 2015 ; 96), qui forge dans ses Rudiments métaphysiques de logique, en opposition avec celui de " système ", le terme $\mathrm{d}^{\text {' } ~ " ~ e x t e ̀ m e ~ " ~}$ (Heidegger 1978 : 269). Celui-ci « déploie au contraire [du système] une force centrifuge qui place l'homme, le monde et le principe toujours à la limite de la désarticulation et de la déhiscence, de la désidentification, voire de leur désintégration, c'est-à-dire de la perte de leur intégrité » (Caye 2015 : 97). L'extème suppose de même la maximalisation de l'être comme puissance d'ouverture illi- 
mitée et comme devenir, afin de permettre ce déploiement absolu d'une liberté qui est moins - faut-il le préciser - la liberté des hommes que celle, quel qu'en soit le prix à payer, de l'être lui-même.

L'histoire du post-structuralisme et de ce qu'on a appelé après Raymond Picard la « Nouvelle critique » (Picard 1965) peut se concevoir comme la théorisation programmatique, dans l'ordre de la poétique, de ce qu'entendait Heidegger à travers son projet de destruction de la métaphysique. On peut rappeler à cet égard le rôle joué par ce dernier dans les milieux intellectuels (français en particulier) à partir des années 50 , grâce notamment au magistère de Jean Beaufret pour la philosophie et de Maurice Blanchot pour la littérature.

Un tel projet est passé en un premier temps par la reprise des acquis de la description sémiologique de Saussure dans la mesure où elle permettait de sortir de la conception humaniste d'une régie du langage par le sujet parlant, mais elle exigeait surtout de la dépasser - c'est-à-dire en fait de la briser. Celle-ci conduit en effet à faire éclater les trois pôles du « système métaphysique » de la modernité : le sujet, privé de sa souveraineté pour être absorbé dans la structure en tant que fonction d'énonciation, le monde tenu en lisière par la coupure sémiologique entre le signe et le référent, l'être mis hors de portée du langage par l'arbitraire du signe. On sait que ces conquêtes ont amené le structuralisme à faire du sens de toute activité à valeur sémiologique la pure et simple projection de la structure de la langue dans le discours, permettant par là-même au passage de déspécifier la littérature et d'évacuer la notion d'œuvre, suspectée de relever d'une forme d'organicité en raison même de sa collusion avec le sujet.

Passer à la logique de l'extrême supposait toutefois d'en finir avec ce qui pouvait encore entraver la pleine libération de la puissance de l'être entendu comme dynamique du devenir, et brider la pleine ouverture du sens. Au dispositif fermé et intemporel d'une structure fonctionnant comme en vase clos, il fallait opposer un déverrouillage des instances et des objets identitaires, dont on sait que la critique de la définition saussurienne du signe a été un enjeu majeur pour des théoriciens tels que Roland Barthes, Julia Kristeva (voir Kristeva 1969) ou Jacques Derrida (voir Derrida 1967 a et b). Je n'ai guère le temps de montrer plus précisément comment le recours à la sémiotique de Peirce ou à la grammaire générative et transformationnelle de Chomsky (voir Chomsky 1969) a été en l'occurrence, chez les deux premiers, le moyen de cette redéfinition jugée nécessaire du modèle linguistique dans le sens d'une circulation indéfiniment ouverte au sein d'un réseau en expansion. Idéologiquement suspect aux yeux de Barthes dans la mesure où il revient à inscrire l'identitaire dans la structure de la langue par le principe de différenciation réciproque des signifiés, le signe saussurien quittait chez lui le statut « scientifique » qui lui était encore conféré en 1966 dans Critique et vérité (voir Barthes 1966), pour devenir en 1971 (voir Barthes 1971) l'instrument d'un impérialisme du sens et de la langue - impérialisme qu'il 
convenait de renverser de l'intérieur par l'ouverture de la littérature, qui ainsi devenait écriture, à la circulation infinie des unités signifiantes et des discours.

Cette neutralisation sémantique des signes, l'infinitisation des parcours possibles du sens qu'elle autorisait, avait par surcroit, du point de vue de ses promoteurs, le triple avantage de libérer pleinement les termes du système au lieu de les bloquer ou de les invalider comme l'avait fait le modèle structuraliste : le sujet devenait instance désirante, subvertissant à la fois son essentialisation humaniste et bourgeoise comme personne et sa réduction fonctionnaliste ; le monde était potentialisé sans limite dans le déploiement de l'écriture qui se substituait à lui en raison de son autonomie, l'un et l'autre pouvant par-là se voir amenés à un état de surfusion révolutionnaire dont la portée était indissociablement esthétique et politique ; l'être enfin devenait une pure dynamique d'indifférenciation, délivrée des contraintes normatives du sens et de la métaphysique de l'unité, et pouvant par là-même justifier une pensée du multiple propre à devenir le paradigme d'un monde capitaliste en voie de dérégulation : on reconnaît là l'opposition - sur le terrain propre de la critique - théorisée par Barthes entre le « lisible » et le « scriptible » (voir Barthes 1970), entre l' « obvie » et l' « obtus » (voir Barthes 1982), ou encore ce que Kristeva a appelé en 1974 « la révolution du langage poétique » (voir Kristeva 1974).

La subversion du signe chez Derrida a emprunté comme on sait d'autres voies pour mettre à mal la métaphysique de l'identité. La destruction du couple identité-altérité, qui dénonce dans le langage l'illusion supposée d'ancrages sémantiques stables et porteurs de pratiques d'oppression (politique, économique, sexuelle), passe chez lui par l'éradication des conditions métaphysiques d'un impérialisme du logos repéré depuis Platon (Derrida « Tel Quel n 32 et 33, 1968), et par l'affaiblissement du signe devenu simulacre et répétition de simulacre - impropre par là-même à désigner un référent et à produire une quelconque stabilité ou un quelconque centrement du sens. On conçoit que ces formalisations poétiques de l' « extème », différentes mais convergentes, qui ont orienté la Nouvelle critique aient pu susciter a contrario les tentatives de reviviscence de l'ontothéologie au sein du « système » ainsi menacé, polarisant fortement le paysage de la théorie littéraire depuis ces cinquante dernières années.

Les antagonismes de l'onto-théologique et du théologico-politique, de l'œuvre et du « désœuvrement » (Blanchot 1955) ${ }^{5}$ apparaissent révélateurs d'une approche métaphysique de la littérature dont l'idée même de destruction de la métaphysique n'est en définitive qu'un aspect. On peut en l'occurrence se demander, en forçant à peine l'hypothèse, si la notion même de littérature telle qu'elle s'est définie depuis un peu plus de deux siècles ne correspond pas spécifiquement à ce qu'a mis en place dans l'ordre poétique la métaphysique du «système », entre une

\footnotetext{
${ }^{5}$ C'est à partir de L'Espace littéraire (Blanchot 1955) que la notion de « désœuvrement » devient centrale dans la réflexion critique de Maurice Blanchot.
} 
caractérisation dominée par la rhétorique et une autre dominée par la décomposition de l'œuvre, et dont le terme d' « écriture » a quelque temps proposé la dénomination. C'est ce que permettent a contrario de mesurer certaines approches logiciennes qui se sont principalement développées dans les pays anglo-saxons, comme celles de sémioticiens comme Nelson Goodman (voir Goodman 2005) et Donald Davidson (voir Davidson 1993), en dialogue avec des logiciens comme Searle, Grice ou Carnap plus qu'avec les critiques littéraires : approches fondées sur les principes fonctionnels d'une économie de l'échange symbolique et d'une exemplification des fonctions cognitives ou émotionnelles du langage ; approches qui, chez Goodman, considèrent la postulation ou la récusation du fondement métaphysique comme une simple donnée formelle, possible mais facultative, d'une construction langagière dont la littérature est un cas particulier, et non pas une exception transcendante comme c'est le cas pour les positions métaphysique ou anti-métaphysique évoquées précédemment. Ni réactionnaire ni subversive, une telle approche relèverait, me semble-t-il plutôt, d'une sorte d'utopie alternative : celle même qu'a fait triompher le paradigme post-métaphysique, fonctionnaliste et libéral dont la fameuse "main invisible » d'Adam Smith a proposé la métaphore économique (voir Smith 2000).

\section{Références bibliographiques}

Auerbach 1969 : E. Auerbach : Mimesis (1946), Paris : Gallimard. Auerbach 1977 : E. Auerbach : Figura, Paris : Gallimard (« tel »). Barthes 1966 : R. Barthes, Critique et vérité, Paris : Seuil. Barthes 1970 : R. Barthes, $S / Z$, Paris : Seuil.

Barthes 1971 : R. Barthes, Sade, Fourier, Loyola, Paris : Seuil.

Barthes 1982 : R. Barthes, L'Obvie et l'obtus, Paris : Seuil.

Blanchot 1955 : M. Blanchot, L'Espace littéraire, Paris : Gallimard.

Bopp 2007 : F. Bopp, Grammaire comparée des langues indo-européennes (1866), Paris : BnF-Gallica.

Caye 2015 : P. Caye, Critique de la destruction créatrice, Paris : Les Belles Lettres, «L'Âne d'or».

Chomsky 1969 : N. Chomsky, Structures syntaxiques [1957], Paris : Seuil.

Court de Gébelin 1773-1782 : Antoine Court de Gébelin, Le Monde primitif analysé et comparé avec le monde moderne (Paris, neuf volumes).

Curtius 1956 : E. R. Curtius, La Littérature européenne et le Moyen Âge latin, Paris : Presses universitaires de France, (réimpr. 1986), 2 vol.

Curtius 1999 : E. R. Curtius, Balzac, Paris : Ēditions des Syrtes.

Derrida 1967 a : J. Derrida, L'Écriture et la différence, Paris : Seuil.

Derrida 1967 b : J. Derrida, La Grammatologie, Paris : Minuit.

Derrida 1968 : Jacques Derrida «La Pharmacie de Platon», Paris : Tel Quel n 32 et 33. 
Diderot 1978 : D. Diderot, Lettre sur les sourds et muets à l'usage de ceux qui entendent et qui parlent [1751], Euvres complètes, tome IV, Paris: Hermann.

Gadamer 1960 : H. G. Gadamer, Vérité et méthode [1960], Paris : Seuil, « L'ordre philosophique ».

Goodman 2005 : N. Goodman, Langages de l'art : Une approche de la théorie des symboles, Paris : Hachette.

Gusdorf 1988 ; G. Gusdorf, Les Origines de l'herméneutique, Paris : Payot.

Hamann 2002 : J. G. Hamann, Aesthetica In Nuce : Métacritique du purisme de la raison pure et autres textes, Paris : Vrin.

Heidegger 1957 : M. Heidegger, «Constitution onto-théologique de la métaphysique », in Questions I et II, Paris, Gallimard.

Heidegger 1977 : M. Heidegger, Schelling. Le traité de 1809 sur la liberté humaine, Paris : Gallimard.

Heidegger 1978 : M. Heidegger, « Metaphysische Anfangsgründe der Logik in Ausgang von Leibniz », in Gesangausgabe, Bd 26, Frankfurt am Main : V. Klostermann.

Jacobi 1946 : F. Jacobi, « Lettre à Fichte » (1799), in Euvres philosophiques (t.f. J.-J. Anstett), Aubier, 1946.

Jauss 1988 : H. R. Jauss, Pour une herméneutique littéraire, Paris : Gallimard.

Kristeva 1969 : J. Kristeva, Semeiotikè, Paris : Seuil.

Kristeva 1974 : J. Kristeva, La Révolution du langage poétique. L'avant-garde à la fin du XIX ${ }^{e}$ siècle, Paris : Seuil.

Leroy 1974 : M. Leroy, « J. Starobinski, Les mots sous les mots. Les anagrammes de Ferdinand de Saussure (compte rendu), Revue belge de Philologie et d'Histoire, $\mathrm{n}^{\circ} 52$, fasc. 3, 659-660.

Molino 1993 : J. Molino, "Port-Royal et la méthode de Sainte-Beuve », in Pour ou contre Sainte-

Beuve : le «Port Royal », Chroniques de Port-Royal, n 42, Genève : Labor et Fides, 175-200.

Picard 1965 ; R. Picard, Nouvelle critique ou nouvelle imposture, Paris : J.J. Pauvert.

Richter 1800 : J.-P. Richter, Clavis fichteana seuLeibgeriana, Erfürt.

Rousseau 1990 : J.-J. Rousseau, Essai sur l'origine des langues, Paris : Gallimard.

Schleiermacher 1994 : F. Schleiermacher, Herméneutique. Pour une logique du discours individuel, Paris, Le Cerf, 1987.

Schlegel 1837 : F. Schlegel, Essai sur la langue et la philosophie des Indiens, Paris : Parent-Desbarres.

Smith 2000 : A. Smith, Recherches sur la nature et les causes de la richesse des nations [1776], Paris : Economica.

Starobinski 1953 : J. Starobinski, Montesquieu par lui-même, Paris : Seuil, «écrivains de toujours $»$.

Starobinski 1961 : J. Starobinski, L'œil vivant, Paris : Gallimard.

Starobinski 1973 : J. Starobinski, 1789. Les Emblèmes de la raison, Paris : Flammarion.

Stendhal 2005 : Stendhal, Racine et Shakespeare [1823-1825], Paris : Kimé. 
Патрик Маро

\section{Два метафизичка модела у књижевној теорији}

Модерна и савремена књижевна критика почива на двама супротстављеним метафизичким моделима : модерност се заправо и развија полазећи од романтичарске концепције „система“ која налаже херменеутичке приступе. Два различито усмерена приступа оспоравају, међутим, само њено начело : први („нова критика“) радикализује систем сводећи га на екстему; а други, као реакција на први,, заговара његово поновно онто-теолошко успостављање.

Кључне речи: екстема, херменеутика, метафизика, модел, онто-теологија, поетика, поструктурализам, систем, теолошко-политичко, теорија. 